\title{
ON THE UNIFORM CONVERGENCE OF FOURIER'S SERIES
}

\author{
By E. W. Hobson.
}

[Received and Read January 10th, 1907.].

The definition of an integral introduced by Lebesgue has led to a notable increase in the scope of Fourier's series. A Fourier's series corresponding to a function $f(x)$ defined in the interval $(-\pi, \pi)$ may be said to exist, whenever the coefficients have a definite meaning, independently of any question as to the convergence of the series. A large class of functions are integrable in accordance with Lebesgue's definition which are not integrable in accordance with the definition of Riemann. For such functions the Fourier's coefficients exist, if the integrals by which they are expressed are interpreted as Lebesgue's integrals. Investigations relating to this extended class of Fourier's series bave been carried out by Lebesgue.* Every summable limited function has a Lebesgue integral, and also some summable functions which are not limited. The latter integrals are always absolutely convergent, in the sense that the absolute values of the functions are also integrable. I have elsewhere shewn that the Lebesgue definition of such an integral is in agreement with the older definitions in cases when those definitions are all applicable. The only integrals which are not covered by Lebesgue's definition are those which are non-absolutely convergent improper integrals. A series in which the coefficients are expressed by such integrals, Lebesgue has proposed to call "generalized Fourier's series." Very little is known about such series, and they will not be referred to in the present communication, which is concerned with the class of Fourier's series in which the coefficients are expressed by Lebesgue integrals, the function employed being either limited or unlimited. Many of the older investigations connected with the theory of Fourier's series were directed to obtaining sufficient conditions for the convergence of the series at a particular point.

* His investigations are contained in two memoirs: "Sur les séries trigonométriques," in the Annales Sc. de l'Ecole Normale, Sér. 3, Vol. xx., 1903; and “Sur la convergence des séries de Fourier," Math. Aunalen, Vol. Lxrv., 1905 ; also in the work Leçons sur les séries trigonométriquces, 1906. 
Later investigations were concerned with conditions that the series might converge uniformly in a whole interval contained in the interval $(-\pi, \pi)$. The present communication is concerned with the latter question, the function to be represented being taken to possess a Lebesgue integral, but not necessarily a Riemann integral, in the interval $(-\pi, \pi)$. A theorem of a general character has been established in $\$ 2$, and the detailed results are deduced from this theorem. It seems not unlikely that this theorem might prove useful in other investigations of a similar character in which the series are not trigonometrical ones, but involve other kinds of periodic functions.

1. The following theorem relating to linear sets of points will be required :-

Let $G$ be a measurable set of points contained in an interval $(a, b)$, and let the set $G$ be enclosed in the interiors of linear intervals forming a finite, or enumerably infinite, set $H$ of non-overlapping intervals, such that $m(H)-m(G)=\eta$, where $\eta$ is a positive number. If $(c, d)$ be any sub-interval whatever, contained in $(a, b)$, and if $G_{1}, H_{1}$ be the parts of $G$ and of $H$ contained in $(c, d)$, then

$$
m\left(H_{1}\right)-m\left(G_{1}\right) \gtrless \eta \text {. }
$$

The term "measurable" is here used in the sense defined by Lebesgue, and the measure of a set $G$ is denoted by $m(G)$.

To prove the theorem, let us assume that, if possible,

$$
m\left(H_{1}\right)-m\left(G_{1}\right)=\eta+a
$$

where $u$ is some positive number. Let the points of $G_{1}$ be enclosed in the interiors of non-overlapping intervals all contained in $(c, d)$, and forming a set $H_{2}$, such that $m\left(H_{2}\right)<m\left(G_{1}\right)+a$; this is possible, since $G_{1}$ is a measurable set. We have then $m\left(H_{2}\right)<m\left(H_{1}\right)-\eta$. Now consider the set of intervals $\bar{H}$ which consists, in $(c, d)$, of the set $H_{2}$, and in the parts of $(a, b)$ not interior to $(c, d)$ of the same intervals that belong to the set $H$.

We have then

$$
m(\bar{H})=m(H)-m\left(H_{1}\right)+m\left(H_{2}\right)<m(H)-\eta<m(G) .
$$

It has thus been shewn that the set $G$ can be enclosed in a set $\bar{H}$ of intervals, such that $m(\bar{H})<m(G)$; but this is impossible, and hence no such positive number as $a$ can exist. Therefore $m\left(H_{1}\right)-m\left(G_{1}\right) \leqslant \eta$. 
2. Let $f(x)$ be a summable function, either limited or unlimited, defined for the interval $(-\pi, \pi)$. In case $f(x)$ be unlimited, it will be assumed to be such that the absolutely convergent integral $\int_{-\pi}^{\pi} f(x) d x$ exists in accordance with Lebesgue's definition. The Fourier's series corresponding to $f(x)$ then exists in the sense that the coefficients in the series all have a definite meaning.

Now let the definition of $f(x)$ be extended so as to apply to values of $x$ lying outside the interval $(-\pi, \pi)$. This extension we make by taking $f(x)$ to be a periodic function of $x$, of period $2 \pi$, except that $f(\pi)$, $f(-\pi)$ may have different values, in which case it is immaterial what values we assign to $f(2 \pi), f(-2 \pi), f(3 \pi), f(-3 \pi), \ldots$.

The following general theorem will now be established :-

The function $f(x)$ being of the character above described, each of the four integrals $\int_{\alpha}^{\beta} f(x \pm 2 z) \chi(z) \sin _{\cos }^{\sin } m d z$, taken through any interval $(\alpha, \beta)$ such that $0 \leqslant \alpha<\beta \leqslant \frac{1}{2} \pi$, converges to the limit zero, as the integer $m$ is increased indefinitely, uniformly for all values of $x$ contained in the interval $(-\pi, \pi)$; the function $\chi(z)$ being any function with limited total fuctuation (ì variation bornée).

More generally, $\sin m z$ or $\cos m z$ may be replaced by $\phi(m z)$, where $\phi(z)$ is any limited summable function of which the integral, taken through any finite interval whatever, is less, in absolute magnitude, than some fixed finite number independent of the particular interval.

First, it will be assumed that $f(x)$ is a limited function. It is sufficient to consider the case of the integral $\int_{\alpha}^{\beta} f(x+2 z) \chi(z) \sin m z d z$; the proof in the case of the other three integrals being precisely similar. Also the substitution of $\phi(m z)$ for $\sin m z$ makes no essential difference in the proof.

Let $U$ and $L$ denote the upper and the lower limits of $f(x+2 z)$, for all the values of $x$ in the interval $(-\pi, \pi)$, and for all values of $z$ in the interval $(\alpha, \beta)$. Let the interval $(L, U)$ be divided into $p$ portions

$$
\left(c_{0}, c_{1}\right),\left(c_{1}, c_{2}\right), \ldots,\left(c_{c_{1-1}}, c_{\eta}\right), \ldots,\left(c_{p-1}, c_{p}\right)
$$

where $c_{0}=L, c_{p}=U$, and $c_{q}-c_{q-1}<\epsilon$, for every value of $q$.

Let the function $f_{1}(x+2 z)$ be defined as follows:-For those values of $x+2 z$ for which $c_{0} \leqslant f(x+2 z)<c_{1}$, let $f_{1}(x+2 z)=c_{0}$; for those values of $x+2 z$ for which $c_{1} \leqslant f(x+2 z)<c_{2}$, let $f_{1}(x+2 z)=c_{1}$; and 
generally let $f_{1}(x+2 z)=c_{q-1}$ for those values of $x+2 z$ for which

$$
c_{q-1} \leqslant f(x+2 z)<c_{q} .
$$

For any particular value of $x$, it way, for example, happen that there are no values of $z$ such that $c_{0} \leqslant f(x+2 z)<c_{1}$ : in that case there are no values of $z$, with the given value of $x$, for which $f_{1}(x+2 z)=c_{0}$.

We have

$$
\left|\int_{a}^{\beta} f(x+2 z) \chi(z) \sin m z d z-\int_{a}^{\beta} f_{1}(x+2 z) \chi(z) \sin m z d z\right|<\epsilon(\beta-\alpha) \bar{\chi}
$$

where $\bar{\chi}$ is the upper limit of $|\chi(z)|$ in the interval $(\alpha, \beta)$; and this holds for all values of $x$ and of $m$.

The integral $\int_{a}^{\beta} f_{1}(x+2 z) \chi(z) \sin m z d z$ is equal to

$$
\sum_{q=0}^{q=p} c_{q} \int_{e_{q}} \chi(z) \sin m z d z
$$

where $e_{\eta}$ is that set of points $z$ at which $c_{q} \leqslant f(x+2 z)<c_{q-1}$; this set $e_{1,}$ depending upon the value of $x$.

In the interval $(-2 \pi, 2 \pi)$ of the variable $x$, let $E_{q}$ be that set of points at each of which $c_{q} \leqslant f(x)<c_{q+1}$. Let the set $E_{q}$ be enclosed in a finite, or enumerably infinite, set $H_{q}$ of non-overlapping intervals such that $m\left(H_{q}\right)-m\left(E_{q}\right)=\eta$. For any fixed value of $x$, the set $e_{q}$ consists of that part of $E_{q}$ which lies in the interval $(x+2 \alpha, x+2 \beta)$ contained in $(-2 \pi, 2 \pi)$. In accordance with the theorem of $\$ 1$, the set of intervals $F_{q_{1}}$ which consists of that part of $H_{q_{1}}$ which lies in $(x+2 \alpha, x+2 \beta)$ is such that $m\left(F_{\eta}\right)-m\left(e_{\eta}\right) \leqslant \eta$. It is to be observed that the number $\eta$ is independent of the value of $x$.

We have also

$$
\left|\int_{e_{q}} \chi(z) \sin m z d z-\int_{P_{q}} \chi(z) \sin m z d z\right|<\bar{\chi} \eta .
$$

Let now the intervals of $H_{1}$ be arranged in diminishing order of length, and let them be denoted by $\gamma_{1}, \gamma_{2}, \gamma_{3}, \ldots$ If $x+2 \alpha$ or $x+2 \beta$ be contained inside an interval of $H_{4}$, we divide that interval into two parts with different indices, each ending at $x+2 \alpha$ or $x+2 \beta$.

We may choose $r$ so that $m\left(H_{11}\right)-\left(\gamma_{1}+\gamma_{2}+\ldots+\gamma_{r}\right)<\eta$. Of the intervals $\gamma_{1}, \gamma_{2}, \gamma_{3}, \ldots$, let those which fall in $(x+2 \alpha, x+2 \beta)$ be $\gamma_{s_{1}}, \gamma_{s_{2}}, \gamma_{s_{3}}, \ldots$ where $s_{1}<s_{2}<s_{3} \ldots$; and let $s_{t}$ be the greatest of these indices which does not exceed $r$. We have then $\gamma_{s_{t+1}}+\gamma_{s_{t+2}}+\ldots<\eta$, and $m\left(F_{t_{l}}\right)-\left(\gamma_{s_{1}}+\gamma_{s_{3}}+\ldots+\gamma_{s_{t}}\right)<\eta ;$ or, denoting by $D_{q}$ the finite set of 
intervals $\gamma_{s_{1}}, \gamma_{s_{3}}, \ldots, \gamma_{s_{t}}$, we have $m\left(F_{q}\right)-m\left(D_{\psi}\right)<\eta$. The number of intervals in the set $D_{q}$ cannot exceed the number $r$, which is independent of the value of $x$.

We now have

$$
\left|\int_{F_{q}} \chi(z) \sin m z d z-\int_{D_{q}} \chi(z) \sin m z d z\right|<\eta \bar{\chi}
$$

The function $\chi(z)$ being a function with limited total fluctuation in the interval $(\alpha, \beta)$, it may be expressed as the difference $\chi_{1}(z)-\chi_{2}(z)$ of trio functions $\chi_{1}(z), \chi_{2}(z)$, each of which is monotone throughout the interval $(\alpha, \beta)$.

The integral $\int_{\mu}^{\lambda} \chi(z) \sin m z d z$, taken through an interval $(\mu, \lambda)$, may by means of the second mean-value theorem be expressed as

$$
\chi_{1}(\mu) \int_{\mu}^{\xi} \sin m z d z+\chi_{1}(\lambda) \int_{\xi}^{\lambda} \sin m z d z-\chi_{2}(\mu) \int_{\mu}^{\xi^{\prime}} \sin m z d z-\chi_{2}(\lambda) \int_{\xi^{\prime}}^{\lambda} \sin m z d z
$$

where $\xi, \hat{\xi}^{\prime}$ are two numbers in the interval $(\mu, \lambda)$. We thus see that

$$
\left|\int_{\mu}^{\lambda} \chi(z) \sin m z d z\right|<\frac{4}{m}\left(\bar{\chi}_{1}+\bar{\chi}_{2}\right)
$$

where $\bar{\chi}_{1}, \bar{\chi}_{2}$ are the upper limits of $\chi_{1}(z), \chi_{2}(z)$ in $(\alpha, \beta)$, the interval $(\mu, \lambda)$ being supposed to be contained in $(\alpha, \beta)$.

We have now

$$
\left|\int_{D_{q}} \chi(z) \sin m z d z\right|<\frac{4}{m}\left(\bar{\chi}_{1}+\bar{\chi}_{2}\right) t<\frac{4}{m}\left(\bar{\chi}_{1}+\bar{\chi}_{2}\right) r
$$

where $t$ is the number of intervals in $D_{y}$, and cannot exceed the number $r$. Combining the inequalities which have been obtained, we find that

$$
\left|\int_{a}^{\beta} f(x+2 z) \chi(z) \sin m z d z\right|<\epsilon(\beta-\alpha) \bar{\chi}+\left(2 \bar{\chi} \eta+\frac{4 r}{m} \overline{\chi^{\prime}}\right) \sum_{\eta=0}^{\prime \prime \prime \prime} c_{y^{\prime \prime}}
$$

where $\bar{\chi}^{\prime}=\bar{\chi}_{1}+\bar{\chi}_{2}$; and this holds for every value of $x$ in $(-\pi, \pi)$. Let us now choose arbitrarily a positive number $\bar{\zeta}$; we can then choose $\epsilon$ so so that $\epsilon(\beta-a) \bar{\chi}<\frac{1}{3} \xi$. Having tixed $\epsilon$ accordingly, and consequently the

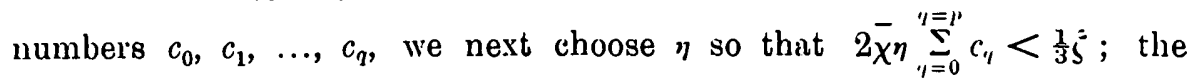
number $r$ is then fixed. We can now choose a value $m_{1}$ of $m$ such that

$$
\frac{4 r}{m} \bar{\chi}, \sum_{\imath=0}^{\imath=p} c_{\eta}<\frac{1}{3} \xi
$$

for $m \geqslant m_{1}$. 
It has now been shewn that, having given a positive number $\xi$, arbitrarily small, a number $m_{1}$ can be so determined that

$$
\left|\int_{a}^{\beta} f(x+2 z) \chi(z) \sin m z d z\right|<\xi,
$$

for $m \geqslant m_{1}$, and for all values of $x$ in the interval $(-\pi, \pi)$. It has therefore been shewn that, when $f(x)$ is a limited summable function,

$$
\int_{a}^{\beta} f(x+2 z) \chi(z) \sin m z d z
$$

converges to the limit zero, as $m$ is indefinitely increased, uniformly for all values of $x$ in the interval $(-\pi, \pi)$, and consequently also for all values of $x$ in any interval $(a, b)$ contained in $(-\pi, \pi)$.

Next, let $f(x)$ be no longer limited, but still integrable in accordance with Lebesgue's definition.

If $\xi$ be an arbitrarily small positive number, a positive number $N$ can be so determined that

$$
\int|f(x)| d x<\frac{1}{2} \xi \mid \bar{\chi},
$$

the integral being taken over that set of points $K_{N}$ in the interval $(-2 \pi, 2 \pi)$, for each of which $|f(x)|>N$. If $k_{N}$ be that part of $K_{N}$ which lies in the interval $(x+2 \alpha, x+2 \beta)$, for any fixed value of $x$ belonging to the interval $(-\pi, \pi)$, we have, a fortiori,

$$
\int_{l_{\mathrm{N}}}|f(x)| d x<\frac{1}{2} \xi / \bar{x} .
$$

Let the function $f_{2}(x+2 z)$ be defined by the specifications

and

$$
\begin{array}{ll}
f_{2}(x+2 z)=f(x+2 z), & \text { if }|f(x+2 z)| \leqslant N, \\
f_{2}(x+2 z)=0, & \text { if }|f(x+2 z)|>N .
\end{array}
$$

Thus $f_{3}(x+2 z)$ vanishes at all the points of the set $k_{N}$, and it is a limited summable function.

We have now

$$
\begin{aligned}
\int_{a}^{\beta} f(x+2 z) \chi(z) \sin m z d z \\
=\int_{k_{N}} f(x+2 z) \chi(z) \sin m z d z+\int_{a}^{\beta} f_{2}(x+2 z) \chi(z) \sin m z d z .
\end{aligned}
$$

By the first part of the theorem, we see that a value $m_{1}$ of $m$ can be determined so that

$$
\left|\int_{a}^{\beta} f_{2}(x+2 z) \chi(z) \sin m z d z\right|<\frac{1}{2} \xi,
$$

for $m \geqslant m_{1}$, and for all values of $x$ in $(-\pi, \pi)$. 
1907.] The UNIForm CONVERGence of Fourier's series.

Also

$$
\left|\int_{k_{N}} f(x+2 z) \chi(z) \sin m z d z\right|<\frac{1}{2} \xi
$$

hence we have shewn that

$$
\left|\int_{\alpha}^{\beta} f(x+2 z) \chi(z) \sin m z d z\right|<\xi,
$$

provided $m \geqslant m_{1}$, for all values of $x$ in $(-\pi, \pi)$. The theorem has therefore been completely established.

3. Some particular cases of the general theorem established in $\$ 2$ will now be considered.

(1) If we consider only the single point $x=0$, we see that, for $a=0$, $\beta=\frac{1}{2} \pi$, and taking $\chi^{(z)}=1$, the integrals

$$
\int_{0}^{3 \pi} f(2 z) \sin _{\cos } m z d z, \quad \int_{0}^{3 \pi} f(-2 z) \sin \cos m z d z
$$

both converge to the limit zero as $m$ is indefinitely increased. Changing $f(2 z)$ into $f(z)$, we see that, if $m=2 n$,

$$
\int_{-\pi}^{\pi} f(z) \frac{\sin }{\cos } n z d z
$$

converges to zero as $n$ is indefinitely increased. The following theorem, which has been proved by Lebesgue, is therefore obtained :-

If $f(x)$ be any summable function, which, if unlimited, still possesses a Lebesgue integral, the coefficients in the corresponding Fourier's series converge to zero as the integer $n$ is indefinitely increased.

(2) It is well known that the condition of convergence of a Fourier's series at a point $x$ where $f(x)$ has an ordinary discontinuity, or is continuous, is that

$$
\int_{0}^{\frac{1}{2} \pi}[f(x+2 z)+f(x-2 z)-f(x+0)-f(x-0)] \frac{\sin m z}{\sin z} d z
$$

should converge to zero, as the odd integer $m$ is indefinitely increased.

Let $\beta=\frac{1}{2} \pi$, and let $\chi(z)=\operatorname{cosec} z$, which is of limited fluctuation in the interval $\left(a, \frac{1}{2} \pi\right)$, if $0<\alpha$. We see then that, if $(a, b)$ be an interval for $x$ in which $f(x)$ is limited, and be also such that $f(a-0)$, $f(b+0)$ are finite, then

$$
\int_{a}^{\frac{1}{2 \pi}}[f(x+2 z)+f(x-2 z)-f(x+0)-f(x-0)] \frac{\sin m z}{\sin z} d z
$$

converges uniformly to zero in the interval $(a, b)$ of $x$. 
For, by the theorem, the two integrals

$$
\int_{a}^{3 z} f(x+2 z) \frac{\sin m z}{\sin z} d z, \quad \int_{a}^{1 \pi} f(x-2 z) \frac{\sin m z}{\sin z} d z
$$

converge uniformly to zero in $(-\pi, \pi)$ and therefore in $(a, b)$; also

$$
|f(x+0)+f(x-0)|
$$

is less for all values of $x$ in $(a, b)$ than some fixed finite number, and $\int_{a}^{t r} \frac{\sin n z}{\sin z} d z$ converges to the limit zero. We therefore see that, in order that a Fourier's series may converge uniformly in an interval $(a, b)$ contained in $(-\pi, \pi)$, it is sufficient that the function be continuous in $(a, b)$, including the end-points, and that, for any arbitrarily small value of the positive number $\alpha$, the integral

$$
\left.\int_{0}^{a}\{f x+2 z)+f(x-2 z)-2 f(x)\right\} \frac{\sin m z}{\sin z} d z
$$

should converge to the limit zero, as $m$ is indefinitely increased, uniformly for all values of $x$ in $(a, b)$.

In this integral, the only functional values of $f(x)$ that are involved are those in the interval $(a-\alpha, b+a)$, provided the values of $x$ in the integrand are confined to those in the interval $(a, b)$. It thus appears that the question whether the Fourier's series converges uniformly in $(a, b)$ depends only upon the nature of the function in the interval $(a-a, b+a)$ which contains $(a, b)$; and the number $a$ is arbitrarily small. We have therefore obtained the following theorem :-

If $(a, b)$ be any interval contained in $(-\pi, \pi)$, such that $f(x)$ is continuous in $(a, b)$, including the end-points $a$ and $b$, then the answer to the question whether the Fourier's series converges uniformly in $(a, b)$, or not, depends only upon the nature of the function $f(x)$ in an interval $\left(a^{\prime}, b^{\prime}\right)$ which includes $(a, b)$ in its interior and exceeds it in length by an arbitrarily small number. The function $f(x)$ may be of any character in the part of $(-\pi, \pi)$ outside $\left(a^{\prime}, b^{\prime}\right)$, subject only to its having a Lebesgue integral in $(-\pi, \pi)$.

This theorem contains, for the theory of uniform convergence, the parallel to the theorem relating to the convergence of the series at a single point, viz., that the convergence or non-convergence of the series at a single point depends only on the nature of the function in an arbitrarily small neighbourhood of that point; being independent of the nature of the function outside that neighbourhood, subject only to the existence 
of the coefficients of the series. This latter theorem was proved by Riemann, for the case of functions satisfying his condition of integrability, and has been proved hy Lebesgue for the case of all summable functions which are integrable in accordance with his definition of integrability. This theorem of Lebesgue is the particular case of the theorem here given, which arises when the interval $(a, b)$ is reduced to a single point $x$.

(3) It is known that, if a function be of limited total Huctuation in $(-\pi, \pi)$, then the corresponding Fourier's series is uniformly convergent in any interval $(a, b)$ which does not contain, either in its interior or at its ends, any point of discontinuity of the function. By applying the theorem obtained in (2), we now obtain the following theorem :-

The function $f(x)$ being summable, and, if unlimited, also integrable, in accordance with Lebesgue's definition; if $\left(a^{\prime}, b^{\prime}\right)$ be any interval contained in $(-\pi, \pi)$, such that $f(x)$ is of limited total fluctuation in $\left(a^{\prime}, b^{\prime}\right)$, then the Fourier's series corresponding to $f(x)$ converges uniformly in any interval $(a, b)$, contained in $\left(a^{\prime}, b^{\prime}\right)$, and such that $f(x)$ is continuous in $(a, b)$, including the end-points.

(4) Let the function $\chi(z)$ be defined by

$$
\chi(0)=0 \quad \text { and } \quad \chi(z)=\frac{1}{z}-\frac{1}{\sin z},
$$

for $z>0$; also let $a=0, \beta=\mu<\frac{1}{2} \pi$; then we see that

$$
\int_{0}^{\mu}[f(x+2 z)+f(x-2 z)-2 f(x)]\left(\frac{1}{z}-\frac{1}{\sin z}\right) \sin m z d z
$$

converges uniformly to zero, as $m$ is indefinitely increased, in any interval $(a, b)$ in which $f(x)$ is limited. It thus appears that, if

$$
\int_{0}^{\mu}[f(x+2 z)+f(x-2 z)-2 f(x)] \frac{\sin m z}{\sin z} d z
$$

converges uniformly in $(a, b)$, so also does

$$
\int_{0}^{\mu}[f(x+2 z)+f(x-2 z)-2 f(x)] \frac{\sin m z}{z} d z .
$$

Therefore, the condition of uniform convergence of the series in an interval $(a, b)$, in which the function is continuous, including the endpoints $a$ and $b$, is that

$$
\int_{0}^{\mu}[f(x+2 z)+f(x-2 z)-2 f(x)] \frac{\sin m z}{z} d z
$$

should converge uniformly to zero in the interval. 
The nunit. , may be taken to be arbitrarily small; in fact, in accordance wivi. $t$ theorem of $\S 2$, if $0<\mu_{1}<\mu$, the integral

$$
\int_{\mu_{1}}^{\mu}[f(x+2 z)+f(x-2 z)-2 f(x)] \frac{\sin m z}{z} d z
$$

converges to zero, as $m$ is indefinitely increased, uniformly in $(a, b)$.

4. We proceed to investigate sufficient conditions for the uniform convergence of the Fourier's series in an interval $(a, b)$ in which the function $f(x)$ is continuous.

Writing $F(z)=f(x+2 z)+f(x-2 z)-2 f(x)$, we express the integral

$$
\int_{0}^{\mu} \frac{F(z)}{z} \sin m z d z
$$

in the form $\left[\int_{0}^{2 \pi / m}+\int_{2 \pi / m}^{3 \pi / m}+\int_{3 \pi / m}^{4 \pi / m}+\ldots+\int_{2 r \pi / m}^{\mu}\right] \frac{F(z)}{z} \sin m z d z$ where $r$ is an integer such that $0 \leqslant \mu-\frac{2 r \pi}{m}<\frac{2 \pi}{m}$.

We assume that $(a, b)$ is contained in an interval $\left(a^{\prime}, b^{\prime}\right)$ in which $f(x)$ is limited; if then we choose $\mu$ to be less than the smaller of the numbers $\frac{1}{2}\left(a-a^{\prime}\right)$, $\frac{1}{2}\left(b^{\prime}-b\right)$, we see that $F(z)$ is limited for all values of $z$ in $(0, \mu)$ and for all values of $x$ in $(a, b)$. We have now

$$
\begin{aligned}
\left|\int_{0}^{2 \pi i m} F(z) \frac{\sin m z}{z} d z\right| & <m \int_{0}^{2 \pi / m}|F(z)| d z \\
& <2 \pi \times \text { upper limit of }|F(z)| \text { in the interval }\left(0, \frac{2 \pi}{m}\right) .
\end{aligned}
$$

Since a continuous function is uniformly continuous, the two functions $f(x+2 z)-f(x), f(x-2 z)-f(x)$ converge to zero, as $z$ converges to zero, uniformly for all values of $x$ in $(a, b)$.

It follows that

$$
\left|\int_{0}^{2 \pi / m} F(z) \frac{\sin m z}{z} d z\right|<\eta_{m}
$$

where $\eta_{n}$ converges to zero, as $m$ is indefinitely increased, and is in. dependent of the value of $x$.

Next, we have

$$
\begin{aligned}
\left|\int_{2 r \pi / m}^{\mu} F(z) \frac{\sin m z}{z} d z\right| & <\frac{\frac{4}{m}}{\mu-\frac{2 \pi}{m}} \times \text { upper limit of }|F(z)| \text { in }\left(\frac{2 r \pi}{m}, \mu\right) \\
& \left.<\frac{\frac{4}{m}}{\mu-\frac{2 \pi}{m}} \times \text { upper limit of }|F(z)| \text { iı }\right\lrcorner(0, \mu)<\eta_{m}^{\prime}
\end{aligned}
$$


where $\eta_{m}^{\prime}$ converges to zero, as $m$ is indefinitely increased, independently of the value of $x$.

The remaining part of the integral may be written in the form

$$
\begin{aligned}
\int_{0}^{\pi^{\prime} m}\left\{\frac{F\left(z+\frac{2 \pi}{m}\right)}{z+\frac{2 \pi}{m}}\right. & -\frac{F\left(z+\frac{3 \pi}{m}\right)}{z+\frac{3 \pi}{m}}+\frac{F\left(z+\frac{4 \pi}{m}\right)}{z+\frac{4 \pi}{m}}-\ldots \\
& \left.+\frac{F\left(z+\frac{\overline{2 r-2} \pi}{m}\right)}{z+\frac{\overline{2 r-} \overline{2} \pi}{m}}-\frac{F\left(z+\frac{2 r-1 \pi}{m}\right)}{z+\frac{2 r-1}{m}}\right\} \sin m z d z,
\end{aligned}
$$

which is less, in absolute value, than

$$
\int_{0}^{\pi}\left|\frac{F\left(\frac{z+2 \pi}{m}\right)}{z+2 \pi}-\frac{F\left(\frac{z+3 \pi}{m}\right)}{z+3 \pi}+\ldots+\frac{F\left(\frac{z+\overline{2 r-2} \pi}{m}\right)}{z+2 r-2 \pi}-\frac{F\left(\frac{z+2 r-1}{m}\right)}{z+2 r-1 \pi}\right| d z_{\text {, }}
$$

which is not greater than

Now

$$
\sum_{s=1}^{s=r-1} \int_{0}^{\pi}\left|\frac{F\left(\frac{z+2 s \pi}{m}\right)}{z+2 s \pi}-\frac{F\left(\frac{z+2 \bar{s}+\overline{1} \pi}{m}\right)}{z+\overline{2 s+1} \pi}\right| d z .
$$

$$
\begin{aligned}
\frac{F\left(\frac{z+2 s \pi}{m}\right)}{z+2 s \pi}- & \frac{F\left(\frac{z+2 s+1 \pi}{m}\right)}{z+\overline{2 s+1} \pi} \\
& =\frac{F\left(\frac{z+2 s \pi}{m}\right)-F\left(\frac{z+\overline{2 s+1} \pi}{m}\right)}{z+2 s+1 \pi}+\pi \frac{F\left(\frac{z+2 s \pi}{m}\right)}{(z+2 s \pi)(z+2 s+1 \pi)}
\end{aligned}
$$

hence

$$
\begin{aligned}
& \left|\frac{F\left(\frac{z+2 s \pi}{m}\right)}{z+2 s \pi}-\frac{F\left(\frac{z+2 \overline{2 s+1} \pi}{m}\right)}{z+2 s \overline{+1} \pi}\right| \\
& \leqslant \frac{\left|F\left(\frac{z+2 s \pi}{m}\right)-F\left(\frac{z+\overline{2 s+1} \pi}{m}\right)\right|}{(2 s+1) \pi}+\frac{1}{2 s(2 s+1) \pi}\left|F\left(\frac{z+2 s \pi}{m}\right)\right| .
\end{aligned}
$$

We now see that the part of the integral to be estimated is, in absolute. value, less than

$$
\left(\frac{1}{3}+\frac{1}{5}+\ldots+\frac{1}{2 r-1}\right) \Delta+\frac{1}{2} \times \text { upper limit of }|F(z)| \text { in }(0, \mu)
$$


where $\Delta$ is the greatest of the numbers $\left|F\left(\frac{z+2 s \pi}{m}\right)-F\left(\frac{z+\overline{2 s+1} \pi}{m}\right)\right|$ for $s=1,2,3, \ldots, r-1$.

The upper limit of $|F(z)|$ or $|f(x+2 z)+f(x-2 z)-2 f(x)|$, for the interval $(0, \mu)$ of $z$, and for all values of $x$ in $(a, b)$, depends upon $\mu$, and is a number $2 u(\mu)$, which may be made as small as we please by taking $\mu$ sufficiently small.

Also

$$
\left(\frac{1}{3}+\frac{1}{5}+\ldots+\frac{1}{2 r-1}\right) \Delta<\left(1+\frac{1}{2}+\frac{1}{3}+\ldots+\frac{1}{2 r}\right) \Delta<\left(C_{r}+\log 2 r\right) \Delta
$$

where $C_{r}$ is a number between 0 and 1 which converges to Mascheroni's constant. Let $D_{n}$ denote the greatest value of the fluctuation of $f(x)$ in a sub-interval of length $\pi / m$ contained in the interval $(a-\mu, b+\mu)$, for all possible positions of such sub-interval; then we have $\Delta \leqslant D_{m}$.

It has now been proved that, for $0<\mu<\frac{1}{2} \pi$,

$$
\begin{aligned}
& \mid \int_{0}^{\mu}[f(x+2 z)+f(x-2 z)-2 f(x)] \frac{\sin m z}{z} d z \mid \\
&<\eta_{m}+\eta_{m}^{\prime}+u(\mu)+D_{m}\left[C_{r}+\log \frac{m}{\pi}+\log \left(\mu-\frac{2 \theta \pi}{m}\right)\right]
\end{aligned}
$$

where $\theta$ is such that $0<\theta<1$, and such that $\mu-\frac{2 r \pi}{m}=\theta \frac{2 \pi}{m}$.

We have now to find a sufficient condition that it be possible, with $\mu$ fixed, to determine a value $\bar{m}$ of $m$, corresponding to an arbitrarily prescribed positive number $\xi$, such that

$$
\left|\int_{0}^{\mu} F(z) \frac{\sin m z}{z} d z\right|<\xi
$$

for $m \geqslant \bar{m}$, and for all values of $x$ in $(a, b)$.

If $\mu_{1}$ be a number such that $0<\mu_{1}<\mu$, we can choose $\mu_{1}$ so small that $u\left(\mu_{1}\right)<\frac{1}{5} \xi$. The number $\mu_{1}$ having been so chosen, we can now choose a value $m_{1}$ of $m$, such that

$$
\left|\int_{\mu_{1}}^{\mu} F(z) \frac{\sin m z}{z} d z\right|<\frac{1}{6} \xi
$$

for $m \geqslant m_{1}$, and for all values of $x$ in $(a, b)$; this follows from the uniform convergence of the integral to the limit zero.

We then have

$$
\left|\int_{0}^{\mu} F(z) \frac{\sin m z}{z} d z\right|<\frac{2}{6} \xi+\eta_{m}+\eta_{m}^{\prime}+D_{n i}\left\{C_{r}+\log \frac{m}{\pi}+\log \left(\mu_{1}-\frac{2 \theta_{1} \pi}{m}\right)\right\},
$$


the numbers $\eta_{m}^{\prime}, D_{m}, \theta_{1}$ now having the values which correspond to $\mu_{1}$ instead of $\mu$. We can now choose $m_{2}$ so that $\eta_{m}<\frac{1}{6} \xi$, for $m \geqslant m_{2}$; also, we can choose $m_{3}$ so that $\eta_{m}^{\prime}<\frac{1}{6} \xi$, for $m \geqslant m_{3}$. Again, since $D_{m}$ converges to zero, as $m$ is indefinitely increased, we can so determine $m$ that $D_{m}\left\{C_{r}+\log \left(\mu_{1}-\frac{\theta_{1} \pi}{m}\right)\right\}<\frac{1}{6} \xi$, for $m \geqslant m_{4}$. Let us now assume that it is possible to so choose $m_{5}$ that $D_{m} \log m / \pi<\frac{1}{6} \xi$, for $m \geqslant m_{5}$. Taking $\bar{m}$ to be the greatest of the numbers $m_{1}, m_{2}, m_{3}, m_{4}, m_{5}$, we now have

$$
\left|\int_{0}^{\mu} F(z) \frac{\sin m z}{z} d z\right|<\xi,
$$

for $m \geqslant \bar{m}$. Therefore, with the assumption made, that $D_{m} \log m / \pi$ converges to zero, as $m$ is indefinitely increased, it has been shewn that the convergence of the Fourier's series in $(a, b)$ is uniform.

The following theorem has accordingly been established :-

It is a sufficient condition for the uniform convergence of the Fourier's series in an interval $(a, b)$, that an interval $\left(a^{\prime}, b^{\prime}\right)$ can be found which encloses $(a, b)$ in its interior, and is such that, if $F_{\beta}$ denote the fluctuation of $f(x)$ in any sub-interval of length $\beta$ contained in $\left(a^{\prime}, b^{\prime}\right), F_{\beta} \log \beta$ converges to zero, uniformly for all such sub-intervals, as $\beta$ is indefinitely diminished.

The condition may also be stated in the form, that it is sufficient that $\{f(x+\beta)-f(x)\} \log \beta$ converge uniformly to zero, as $\beta$ is indefinitely diminished, for all values of $x$, such that $x$ and $x+\beta$ are in the interval $\left(a^{\prime}, b^{\prime}\right)$ which encloses $(a, b)$.

This is seen by referring back to the foregoing proof, where the difference of functional values at the ends of an interval $\beta$ was replaced by the fluctuation in that interval.

The condition is satisfied if $|f(x \pm \beta)-f(x)| \leqslant C \beta^{k}$, where $C$ and $k$ are positive numbers, for all values of $\beta$ less than some fixed value $\beta_{1}$, for every value of $x$ such that $x, x \pm \beta$ are in $\left(a^{\prime}, b^{\prime}\right)$, the numbers $C$ and $k$ being independent of $x$.

The particular cases of these theorems which arise when $(a, b)$ is reduced to a single point are known sufficient conditions of convergence of the series at a single point, due to Lipschitz.

The function $f(x)$ has throughout been assumed to be restricted in the whole interval $(-\pi, \pi)$, only so far that it is either a limited summable function, or an unlimited summable function which possesses a Lebesgue integral in $(-\pi, \pi)$. 
5. Sufficient conditions for the uniform convergence of the series in an interval $(a, b)$ may also be obtained in the following simple manner:If $0<\mu_{1}<\mu$, we have

$$
\left|\int_{0}^{\mu} \frac{F(z)}{z} \sin m z d z\right| \leqslant\left|\int_{0}^{\mu_{1}} \frac{F(z)}{z} \sin m z d z\right|+\left|\int_{\mu_{1}}^{\mu} \frac{F(z)}{z} \sin m z d z\right| .
$$

Let it now be assumed that, for every value of $x$ in $(a, b), \int_{0}^{\mu_{1}} \frac{F(z)}{z} d z$ exists, and that $\int_{0}^{\mu_{1}}\left|\frac{F(z)}{z}\right| d z$ converges to the limit zero, as $\mu_{!}$is indefinitely diminished, uniformly for all values of $x$ in $(a, b)$.

We have then

$$
\left|\int_{0}^{\mu_{1}} \frac{F(z)}{z} \sin m z d z\right|<\int_{0}^{\mu_{1}}\left|\frac{F(z)}{z}\right| d z
$$

The number $\mu_{1}$ can now be chosen so small that, if $\xi$ be an arbitrarily fixed positive number, the inequality

$$
\int_{0}^{\mu_{1}}\left|\frac{F(z)}{z}\right| d z<\frac{1}{2} \xi
$$

is satisfied for this value of $\mu_{1}$, and for every value of $x$ in $(a, b)$. The number $\mu_{1}$ having been so fixed, we can fix a value $m_{1}$ of $m$ such that

$$
\left|\int_{\mu_{1}}^{\mu} \frac{F(z)}{z} \sin m z d z\right|<\frac{1}{2} \zeta
$$

for $m \geqslant m_{1}$, and for every value of $x$ in $(a, b)$. We have then

$$
\left|\int_{0}^{\mu} \frac{F(z)}{z} \sin m z d z\right|<\xi
$$

for $m \geqslant m_{1}$, and for every value of $x$ in $(a, b)$.

The following theorem has therefore been established :-

It is a sufficient condition for the uniform convergence of the Fourier's series in an interval $(a, b)$ contained in an interval $\left(a^{\prime}, b^{\prime}\right)$ in which $f(x)$ is limited that

$$
\int_{0}^{\mu_{1}}\left|\frac{f(x+2 z)+f(x-2 z)-2 f(x)}{z}\right| d z
$$

should exist for all values of $x$ in $(a, b)$, and converge to zero as $\mu_{1}$ is indefinitely diminished, uniformly for all values of $x$ in $(a, b)$. The condition will be satisfied if the two integrals

$$
\int_{0}^{\mu_{1}}\left|\frac{f(x+2 z)-f(x)}{z}\right| d z, \quad \int_{0}^{\mu_{1}}\left|\frac{f(x-2 z)-f(x)}{z}\right| d z
$$

both exist and are uniformly convergent. 
In particular, the series is uniformly convergent in any interval $(a, b)$ in which one of the four derivatives, and therefore each of the other three derivatives, is limited, the end-points $a$ and $b$ being included. A special case is that in which $f(x)$ has a limited differential coefficient throughout $(a, b)$, including $a$ and $b$.

The condition is satisfied if $|f(x \pm \beta)-f(x)|<C \beta^{k}$, for all values of $x$ in $(a, b)$, and for all values of $\beta$ less than some tixed number; where $C$ and $k$ are fixed positive numbers independent of $x$. This condition is, at least in form, slightly simpler than the similar condition given in $\S 4$. If $(a, b)$ be reduced to a single point, it at once becomes the known condition of convergence at $x$, that $|f(x \pm \beta)-f(x)|<C \beta^{r}$. 